\title{
Comprehensive Simulation of Cooperative Robotic System for Advanced Composite Manufacturing: A Case Study
}

\author{
Manman YANG ${ }^{\mathrm{a}}$, Leijian YUa, Cuebong $\mathrm{WONG}^{\mathrm{b}}, \mathrm{Carmelo} \mathrm{MINEO}^{\mathrm{c}}, \mathrm{Erfu}^{\mathrm{a}} \mathrm{YNG}^{\mathrm{a}}$, \\ Iain BOMPHRAY ${ }^{\mathrm{d}}$, Ruoyu HUANG ${ }^{\mathrm{d}}$, Scott BRADY \\ ${ }^{a}$ Department of Design, Manufacture \& Engineering Management, University of \\ Strathclyde, Glasgow G1 1XJ, UK \\ ${ }^{\mathrm{b}}$ National Nuclear Laboratory Ltd. Havelock Rd, CA14 3 YQ Workington, UK \\ ${ }^{\mathrm{c}}$ Department of Engineering, University of Palermo, Viale delle Scienze, Edificio 8, \\ 90128 Palermo, Italy \\ ${ }^{\mathrm{d}}$ Lightweight Manufacturing Centre, University of Strathclyde, Glasgow G1 1XJ, UK \\ e Advanced Forming Research Centre, University of Strathclyde, Glasgow G1 1XJ, UK
}

\begin{abstract}
Composite materials are widely used because of their light weight and high strength properties. They are typically made up of multi-directional layers of high strength fibres, connected by a resin. The manufacturing of composite parts is complex, time-consuming and prone to errors. This work investigates the use of robotics in the field of composite material manufacturing, which has not been well investigated to date (particularly in simulation). Effective autonomous material transportation, accurate localization and limited material deformation during robotic grasping are required for optimum placement and lay-up. In this paper, a simulation of a proposed cooperative robotic system, which integrates an autonomous mobile robot with a fixed-base manipulator, is presented. An approach based on machine vision is adopted to accurately track the position and orientation of the fibre plies. A simulation platform with a built-in physics engine is used to simulate material deformation under gravity and external forces. This allows realistic simulation of robotic manipulation for raw materials. The results demonstrate promising features of the proposed system. A root mean square error of $9.00 \mathrm{~mm}$ for the estimation of the raw material position and 0.05 degrees for the fibre orientation detection encourages further research for developing the proposed robotic manufacturing system.
\end{abstract}

Keywords. Robotics, composite manufacturing, simulation

\section{Introduction}

Composite materials are attracting more and more attention because of their outstanding performance, especially their small weight-to-strength ratio [1]. They are typically made up of multi-directional layers of high-strength fibres, connected by a plastic resin. The manufacturing of composite parts is complex, time-consuming and prone to errors [2]. Currently, it is mostly a manually driven process. This work investigates the use of robotics in the field of composite material manufacturing. Industry 4.0, the current innovation trend that boosts the development and introduction of intelligent robotic 
systems and new logistic solutions for the handling of materials in factories, is transforming manufacturing in many sectors. In recent years, there has been an increasing demand for the phasing in of robotic solutions for greater flexibility and autonomy. Unfortunately, composite manufacturing has benefitted little from this technology shift. Simulation is an effective and straightforward way to validate proposed methods and algorithms. This is also true for advanced composite manufacturing, which comprises a series of large equipment and intricate laborious processes [3].

In recent years, previous works have investigated the possibility of using robotic systems in advanced composite manufacturing, due to the expected flexibility of robotic manufacturing strategies. Schuster et al. investigated robotic manipulators able to pick and place large composite plies, minimizing placement wrinkles [4, 5]. A similar approach was adopted by Deden et al., who also addressed collision-free robotic path planning requirements [6]. However, cooperative/hybrid robotic systems, comprising mobile robot platforms and fixed robot manipulators have been less researched in advance composite manufacturing.

This paper presents a case study that demonstrates how a Cooperative Mobile Robot and Manipulator System (Co-MRMS) can be utilized in advanced composite manufacturing for material transport and pick-and-place operations. The integrated robotic system framework is described in section 2. Modelling methods, including the modelling of deformable materials and the simulation of the machine vision module, are demonstrated in section 3. Simulation results and their analysis are discussed in section 4 and conclusions are presented in section 5 .

\section{Integrated robotic system framework}

Co-MRMS integrates an autonomous mobile robot with a fixed-base manipulator. The mobile robot is responsible for transporting the composite material. With the help of a vision module, the position and orientation of the raw material is sent to the fixed base robot manipulator. This latter robot is used for grasping each fiber ply and placing it correctly according to the designed lay-up manufacturing specifications. The simulation framework for Co-MRMS is presented in Figure 1. Robotic path planning for both the mobile and fixed robot platforms were implemented in MATLAB. A camera was mounted on the end-effector of the fixed robotic manipulator to support a machine vision solution. OpenCV was used for image processing. Simulations of the full system were generated in CoppeliaSim.

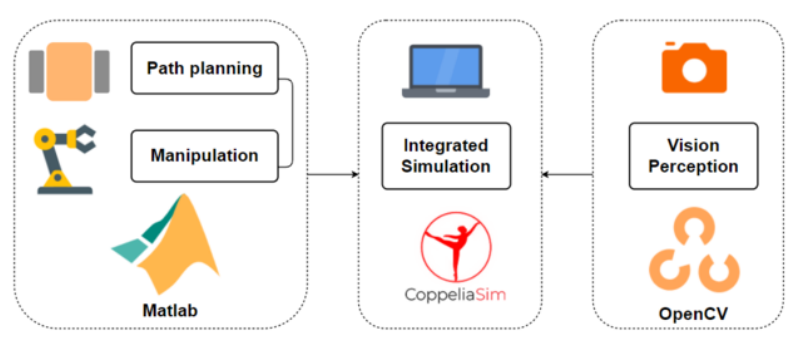

Figure 1. Co-MRMS architecture flowchart.

The simulation environment in CoppeliaSim and the view of composite material placed on mobile robot and mould are presented in Figure 2. The simulation framework 
can be divided into two stages: autonomous raw material transportation and composite material handling. In the first stage, the mobile robot deploys a collision-free path obtained by using a modified variant of the Bi-directional RRT* path planning algorithm based on [7], which enables the robot to autonomously navigate from any start location to a goal location. Once the mobile robot arrives at the goal, the second stage begins. Odometry data of the mobile robot is transmitted to the CoppeliaSim simulation environment through the use of the Robot Operating System (ROS). Following the reception of the estimated material position, the fixed robotic manipulator moves its endeffector, which carries an arrangement of suction cups for grasping operations, from the home position to a standby position above the material. The machine vision module is activated once such position is reached and an image of the scene is captured through the camera. The image is processed in OpenCV to gain a more accurate estimation of the material position and to compute the fibre orientation. Such position is transmitted to CoppeliaSim through UDP (User Datagram Protocol). The fixed robot manipulator adjusts the end-effector position and orientation, according to the received vision data. The end-effector approaches the deformable material, grasps the material and transfers it to the mould. Finally, the robot returns to its home position.

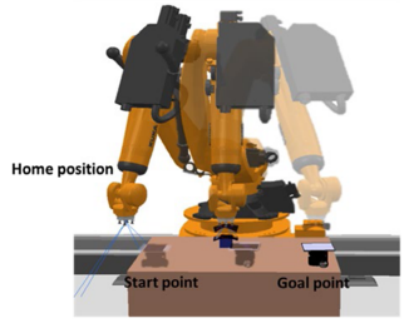

(a)

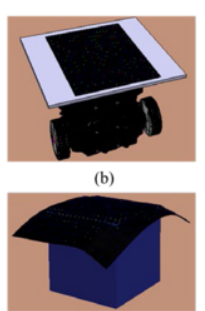

(c)

Figure 2. (a) Simulation environment in CoppeliaSim. (b) and (c) are composite material's placement on mobile robot and mould respectively.

\section{Simulation method}

\subsection{Deformable Object Modelling and Suction Cup End Effector}

The modelling of the deformable composite material within CoppeliaSim was inspired by the particle-based method described in [8] and consists of representing a sheet material as an array of primitive cuboids. For each individual cuboid within the model, up to four dummy links were attached to the shape at a fixed distance from the outer edges. A simple 3x3 example of a composite material model, consisting of individual cuboids and associated dummies. Dummies between adjacent cuboids were linked by dynamic overlapping constraints to emulate stretching and bending behaviours of the material. These dynamic constraints constrain the relative motion between linked dummies.

One important factor in modelling deformable objects is the stiffness level of the material. The adopted method for modelling deformable objects enables this stiffness to be adjusted by tweaking two different types of model parameters: the $\mathrm{x}$ and $\mathrm{y}$ dimensions of each individual primitive cuboid (represented by $\mathrm{L}_{\mathrm{x}}$ and $\mathrm{L}_{\mathrm{y}}$ respectively) and the principle moments of inertia along the $\mathrm{x}, \mathrm{y}$ and $\mathrm{z}$ axes (represented by $\mathrm{I}_{\mathrm{x}}, \mathrm{I}_{\mathrm{y}}$ and $\mathrm{I}_{\mathrm{z}}$ respectively). The primitive cuboid dimensions and principle moments of inertia are 
presented in Table 1, where the height of each cuboid represents the thickness of composite material.

Table 1. Model parameters for composite material deformation.

\begin{tabular}{c|c}
\hline Model parameters & Value \\
\hline Principle moment of inertia $\mathrm{I}_{\mathbf{x}} / \mathrm{mm}^{2}$ & 1.25 \\
\hline Principle moment of inertia $\mathrm{I}_{\mathbf{y}} / \mathrm{mm}^{2}$ & 4.25 \\
\hline Principle moment of inertia $\mathrm{I}_{\mathbf{z}} / \mathrm{mm}^{2}$ & 15.00 \\
\hline Length $\mathrm{L}_{\mathbf{x}} / \mathrm{mm}$ & 12.00 \\
\hline Width $\mathrm{L}_{\mathbf{y}} / \mathrm{mm}$ & 6.00 \\
\hline Height $\mathrm{L}_{\mathbf{z}} / \mathrm{mm}$ & 1.00
\end{tabular}

To simulate the grasping of this material model, a vacuum gripper, comprising of an arrangement of suction cups, was developed in CoppeliaSim. The suction cup model available in CoppeliaSim's standard library are only compatible with rigid bodies as it simulates a grasp using a single contact point. This results in a sagging effect when used for grasping the non-rigid material model developed in this work. To circumvent this, a modified suction cup model was developed to ensure contact is made between all cuboids that lie within the grasp region of a suction cup during grasp operations. This modified suction cup provides a robust simulation component for quick evaluation of different vacuum gripper designs.

\subsection{Material localisation and Fibre direction identification}

\subsubsection{Material localisation:}

In order to localise the position of the material, its geometry information plays a key role. As shown in Figure 3, images captured by the simulated vision sensor are converted to greyscale images to reduce unrelated information. Afterwards, a Gaussian blur filter [9] is adopted to smooth the data. The image is then transformed to a binary image and postprocessed through morphological operations. Finally, the pose information of the material is obtained through analysing its shape and area.

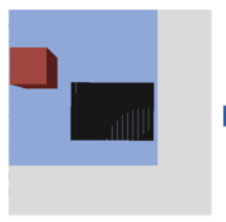

Original image

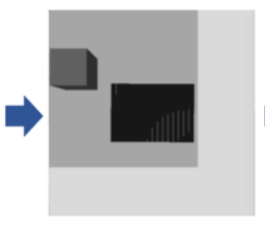

Blurred Grey image

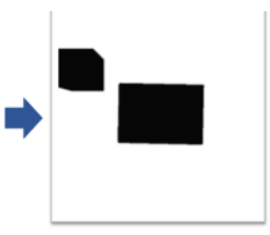

Post-processed Binary image

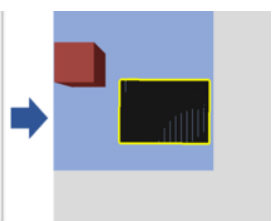

Result

Figure 3. Image processing workflow.

When the relative height between the camera and material is fixed, the scale of image pixels and real-world distance can be calculated in accordance with the pinhole camera theory [10]. Therefore, the real distance between the centre of the camera and the surface of the part is inferred. 


\subsubsection{Fibre direction detection:}

With a series of pre-processing operations (Gaussian blur and morphological operations), the reflections produced by the fibres are extracted from the material texture. As the fibres are not perfectively straight, calculating the slope and intercept of the fitting lines is not straightforward. To overcome this, all detected fibres in the image space are transformed into the Hough space. Each line in the image space is represented by a point in the Hough space. As shown in (1), the formula of the line in the image space is:

$$
\rho^{\prime}=x \cos \left(\theta^{\prime}\right)+y \sin \left(\theta^{\prime}\right)
$$

Where $\rho^{\prime}$ is the distance from the origin to the line, and $\theta^{\prime}$ is the angle between the positive $\mathrm{x}$-axis and the normal to the line.

\section{Simulation and results}

To verify the feasibility of the proposed Co-MRMS in fulfilling the transfer-detect-pickplace task in advanced composite manufacturing (see in section 2), a KUKA KR90 R3100 industrial fixed-base manipulator, and a Turtlebot3 Burger differential drive mobile robot were modelled in CoppeliaSim. To detect and grasp the material, an integrated camera unit and four suction cups were modelled on the KUKA KR90 endeffector. Two experiments were conducted in the simulation environment to assess the theoretical accuracy of the machine vision system, which reflects the achievable accuracy of Co-MRMS. The first experiment corresponds to material localization. The mobile robot drove autonomously to a randomly generated goal within the workspace of the manipulator and Gaussian noise (Mean $=100 \mathrm{~mm}$, Standard deviation $=70 \mathrm{~mm}$ ) was introduced into the estimated material position to simulate the odometry error in real environments. This test was repeated 10 times for the evaluation of vision error and odometry error in material localization. The Mean Absolute Error (MAE) and the Root Mean Squared Error (RMSE) against the ground truth are shown in Table 2. The MAE and RMSE of odometry were $158.48 \mathrm{~mm}$ and $121.21 \mathrm{~mm}$ respectively while the MAE and RMSE of the vision system were $11.53 \mathrm{~mm}$ and $9.00 \mathrm{~mm}$ respectively. These results clearly show that machine vision can drastically improve the accuracy of the estimated material position. The second experiment evaluates the performance of fibre orientation detection. The camera was kept static while the orientation of the composite material was varied by $10^{\circ}$ increments within the range of $\left[0^{\circ}, 180^{\circ}\right]$. These were compared with the ground truth extracted from the simulation to determine the accuracy of the system. The $\mathrm{MAE}$ and RMSE for fibre orientation detection error were found to be $-0.023^{\circ}$ and $0.048^{\circ}$, respectively, as shown in Table 2. These findings demonstrate that the Co-MRMS has the potential to achieve high accuracy detection in controlled environments with diffused lighting and the absence of reflections.

Table 2. Material localization and fibre direction detection error.

\begin{tabular}{l|l|c|c}
\hline \multicolumn{2}{l|}{} & MAE & RMSE \\
\hline \multirow{2}{*}{ Material localization } & Odometry/mm & 158.48 & 121.21 \\
\cline { 2 - 4 } & Vision/mm & 11.53 & 9.00 \\
\hline Fibre direction detection & Vision/degree & -0.023 & 0.048 \\
\hline
\end{tabular}




\section{Conclusions}

This work proposed a Cooperative Mobile Robot and Manipulator System (CoMRMS), which provides a promising strategy for autonomous transfer-detect-pick-place task in advance composite manufacturing. The system is validated in simulation. The full simulation comprises a machine vision module, an autonomous mobile robot, a grasping mechanism, a deformable object model (representing a fibre ply for composite manufacturing) and a fixed robotic manipulator. Reorientation and placement of the composite ply was simulated in CoppeliaSim. The deformable ply object was modelled by considering the realistic stiffness values of composite plies. The conducted simulation tests proved the feasibility and effectiveness of the proposed robotic system. As validated in multiple simulations, the MAE and RMSE for material localization can be drastically reduced by using machine vision to correct for odometry error. The fibre orientation can also be accurately estimated within suitable tolerances through the use of machine vision techniques. This makes Co-MRMS a good candidate system for improving the autonomy of composite manufacturing in the Industry 4.0 era.

\section{Acknowledgements}

This research is supported in part by the Advanced Forming Research Centre (AFRC) and Lightweight Manufacturing Centre (LMC) under the Route to Impact Program 2019-2020 funded by Innovate UK High Value Manufacturing Catapult [grant no.: AFRC_CATP_1469_R2I-Academy].

\section{References}

[1] R. M. Christensen, Mechanics of composite materials. Courier Corporation, 2012.

[2] S. V. Hoa, Principles of the manufacturing of composite materials. DEStech Publications, Inc, 2009.

[3] F. C. Campbell Jr, Manufacturing processes for advanced composites. elsevier, 2003.

[4] A. Schuster et al., "Simulation based draping of dry carbon fibre textiles with cooperating robots," Procedia Manufacturing, vol. 38, pp. 505-512, 2019.

[5] A. Schuster, M. Kupke, and L. Larsen, "Autonomous manufacturing of composite parts by a multi-robot system," Procedia Manufacturing, vol. 11, pp. 249-255, 2017.

[6] D. Deden, C. Frommel, R. Glück, L.-C. Larsen, M. Malecha, and A. Schuster, "Towards a fully automated process chain for the lay-up of large carbon dry-fibre cut pieces using cooperating robots," SAMPE Europe 2019, 2019.

[7] C. Wong, E. Yang, Y. Xiu, and D. Gu, "Optimal Path Planning Based on a Multi-Tree T-RRT* Approach for Robotic Task Planning in Continuous Cost Spaces," presented at the 2018 12th France-Japan and 10th Europe-Asia Congress on Mechatronics, Tsu, Japan, 2018.

[8] D. E. Breen, D. H. House, and M. J. Wozny, "A particle-based model for simulating the draping behavior of woven cloth," Textile Research Journal, vol. 64, no. 11, pp. 663-685, 1994.

[9] J. Flusser, S. Farokhi, C. Höschl, T. Suk, B. Zitová, and M. Pedone, "Recognition of images degraded by Gaussian blur," IEEE transactions on Image Processing, vol. 25, no. 2, pp. 790-806, 2015.

[10] M. Young, "Pinhole optics," Applied Optics, vol. 10, no. 12, pp. 2763-2767, 1971. 